${ }^{1}$ Universidad Santo Tomás, Viña del Mar, Chile.

Universidad de Almería, España.

${ }^{3}$ Universidad de Granada, España.

${ }^{4}$ Servicio de Kinesiología CESFAM Dr.

Boris Soler, Melipilla, Santiago de Chile.

aProfesor Doctor por la UGR, Escuela

de Kinesiología.

bProfesor Doctor por la UAL, Área de Fisioterapia.

'Profesor Doctor por la UGR, Área de Didáctica de la Expresión Corporal. ${ }^{\mathrm{d} K i n e s i o ́ l o g o . ~}$

Los autores declararon no tener conflictos de intereses.

Los autores declararon haber tenido

la siguiente participación en el

manuscrito: CE Jiménez: concepción

y diseño del estudio; recolección/

obtención de resultados; redacción

del manuscrito; R. Fernández: revisión

crítica del manuscrito; asesoría

técnica o administrativa; aprobación de la versión final; F. Zurita: análisis e interpretación de datos; asesoría estadística; aprobación de la versión

final; D. Linares: concepción y diseño del trabajo, revisión crítica del manuscrito; A. Farías: aporte de pacientes o material de estudio; asesoría técnica o administrativa.

Recibido el 16 de enero de 2013, aceptado el 10 de abril de 2014

Correspondencia a:

Sr. Christian Edgardo Jiménez Schyke Universidad Santo Tomás, 1 Norte 3041, Viña del Mar, Chile. cjimenezs@santotomas.cl

\section{Programas de Educación en Salud y Entrenamiento de la Fuerza en adultos mayores con artrosis de cadera leve a moderada}

\author{
CHRISTIAN EDGARDO JIMÉNEZ S. 1,a, RUBÉN FERNÁNDEZ G. 2,b, \\ FÉLIX ZURITA O., ${ }^{3, \mathrm{c}}$, DANIEL LINARES G. ${ }^{3, \mathrm{c}}$, ARIEL FARÍAS M. ${ }^{4, \mathrm{~d}}$
}

\section{Effects of education and strength training on functional tests among older people with osteoarthritis}

Background: Hip and knee osteoarthritis are important causes of pain and disability among older people. Education and strength training can alleviate symptoms and avoid functional deterioration. Aim: To assess muscle strength, fall risk and quality of life of older people with osteoarthritis and the effects of physiotherapy education and strength training on these variables. Material and Methods: Thirty participants aged $78 \pm 5$ years (63\% women) were randomly assigned to receive physiotherapy (Controls), physiotherapy plus education (Group 1) and physiotherapy plus strength training (group 2). At baseline and after 16 weeks of intervention, patients were evaluated with the Senior Fitness Test, Timed Up and Go and Quality of Life score short form (SF-36). Results: During the intervention period, Senior Fitness Test and Timed Up and Go scores improved in all groups and SF-36 did not change. The improvement in Senior Fitness Test and Timed Up and Go was more marked in Groups 1 and 2 than in the control group. Conclusions: Education and strength training improve functional tests among older people with osteoarthritis.

(Rev Med Chile 2014; 142: 436-442)

Key words: Frail elderly; Hip; Muscle Strength; Physical education and training.
41 laumento de las expectativas de vida a nivel mundial ha generado cambios estructurales en la dinámica de las poblaciones actuales. Este hecho inédito de la sociedad actual no refleja más que el éxito alcanzado en el control de la morbilidad y su comprensión en políticas de salud pública que consideran a la salud como un derecho inherente del ser humano ${ }^{1}$. El número de personas mayores de 60 años aumentó de 400 millones en la década de 1950-59 a 700 millones en la década de 1990-99, estimándose que en el año 2025 existirán 1.200 millones de ancianos ${ }^{2}$. Así podremos caracterizar al siglo XXI como el siglo del envejecimiento de la población, instaurando un importante desafío en la atención médica y social ${ }^{3}$. Destacada importancia cobra la evaluación geriátrica integral y de la funcionalidad como elementos sensibles para la detección de adultos mayores saludables ${ }^{4}$. Así, la práctica permanente de ejercicio físico se instaura como una 
prioridad de fomento en salud pública con el fin de prevenir las manifestaciones de enfermedades crónicas degenerativas no transmisibles ${ }^{5}$, como la artrosis, fatiga (por inactividad física), debilidad, alteraciones músculo esqueléticas y cardiovasculares ${ }^{6}$, que aumentan el riesgo de caídas ${ }^{7}$. Otros factores esenciales en la evolución negativa del adulto mayor son el sedentarismo, atrofia muscular, sarcopenia o alteración del equilibrio, que en ocasiones se asocian con dolor y limitación de los rangos articulares.

Las artralgias secundarias a artrosis son la fuente más común de sintomatología y de limitación funcional en el adulto mayor ${ }^{8-9}$. La artrosis de cadera es un cuadro clínico incapacitante y de alta prevalencia en adultos mayores, en Chi$\mathrm{le}^{10}$. Los pacientes que sufren de esta patología, frecuentemente no encuentran solución en el sistema público de salud por diferentes causas. Entre ellas destacamos el prolongado tiempo que deben esperar para el diagnóstico de un especialista, lo cual produce detecciones tardías con el consecuente avance en la degeneración articular y sintomatología dolorosa ${ }^{11}$.

La educación para la salud en estos pacientes es una estrategia que resulta exitosa, entregando un beneficio mayor al tratamiento clásico con antiinflamatorios no esteroidales ${ }^{11-13}$, pero igual importancia tiene el ejercicio terapéutico de fortalecimiento ${ }^{14-16}$ y la fisioterapia ${ }^{17-20}$. En la medida que podamos generar intervenciones efectivas coadyuvantes para el tratamiento de la artrosis leve a moderada, (puntuada entre 0 y 3 ) de acuerdo a los criterios radiológicos de la Sociedad Española de Reumatología ${ }^{21}$, en adultos mayores podremos prolongar el tiempo de espera hacia la intervención de una endoprótesis total.

A partir de estas directrices, y teniendo en cuenta el escaso número de estudios científicos relacionados con la temática que aborden conjuntamente todos los parámetros que aquí se presentan, y remarcando además el efecto que pueden tener los programas de educación para la salud y mejora de la fuerza en la calidad de vida en pacientes con artrosis de cadera, hemos planteado este estudio cuyos objetivos son: 1. Evaluar la fuerza muscular funcional, riesgo de caídas y calidad de vida en adultos mayores con artrosis leve a moderada de cadera; y 2. Valorar los efectos sumatorios de un programa de fisioterapia, educación en salud y fortalecimiento muscular en los adultos mayores.

\section{Pacientes y Métodos}

\section{a) Pacientes}

Estudio cuasi experimental en que participaron 30 adultos mayores de 65 años $(\mathrm{M}=77,7$ años; $\mathrm{DE}=4,94), 19$ mujeres $(63,3 \%)$ y 11 hombres $(36,7 \%)$.

Estas personas asistían a los Centros de Salud de Atención Primaria de la Comuna de Melipilla, Chile, entre marzo y junio de 2011 y fueron derivados al Servicio de Rehabilitación con diagnóstico médico y radiológico de artrosis de cadera leve a moderada. Los criterios de inclusión fueron: Personas autovalentes sin patología crónica descompensada, con capacidad para recorrer una distancia de 100 metros sin ayudas técnicas y con nivel cognitivo que permitiera la comprensión de órdenes simples.

Los 30 pacientes fueron distribuidos en forma aleatoria a tres grupos de 10 personas.

- Grupo Control Activo, quienes recibieron tratamiento fisioterapéutico (FST) basado en ultratermia y TENS, exclusivamente.

- Grupo 1, a quienes se agregó al tratamiento fisioterápico un programa de Educación en Salud (ES).

- Grupo 2, donde los participantes recibieron FST más ES y un programa de entrenamiento de la fuerza $(\mathrm{EF})$.

\section{b) Instrumentos}

Se emplearon instrumentos validados para evaluar las variables fuerza muscular, riesgo de caídas y calidad de vida.

- Para la valoración de la fuerza muscular funcional, se utilizó el número de repeticiones en la prueba pararse y sentarse, obtenido del "Senior Fitness Test" 22 , que valora la fuerza en las extremidades inferiores, donde el participante se sitúa sentado en una silla con los brazos cruzados y manos en hombros, debiendo realizar la mayor cantidad de repeticiones posibles, en 30 segundos, contando el ciclo completo, esto es puesta de pie y vuelta a posición de sentado.

- El riesgo de caídas fue valorado a través de la prueba "Timed Up and Go", descrita en el Examen de Medicina Preventiva del Adulto Mayor $^{23}$. El paciente, sentado en una silla, se pone de pie sin ayuda técnica, debe recorrer una distancia de 3 metros y volver a sentarse con el dorso apoyado en el respaldo. El tiempo 
es cronometrado, considerando un tiempo mayor a 10 segundos como riesgo de caída.

- Para la determinación de la calidad de vida relacionada con la salud, se utilizó el cuestionario autoadministrado "Short Form 36 (SF-36)" versión 2.0 , validado para población nacional chilena ${ }^{24}$, que considera un conjunto de preguntas agrupadas en componentes físicos y componentes mentales, cada una valorada según escala tipo Likert con valores de 0 a 100 (de peor a mejor estado de salud).

\section{c) Procedimiento}

Se solicitó la colaboración de los participantes mediante consentimiento informado de los mismos y siguiendo los protocolos aprobados en la Declaración de Helsinki, con la autorización del comité ético constituido en el Centro de Salud Familiar Dr. Boris Soler, no encontrando ningún participante que se negase a participar en este estudio. Las pruebas se realizaron durante los meses de agosto a diciembre de 2011. Se había realizado una valoración previa, siguiendo los pasos del protocolo de aplicación, e indicándoles a los participantes el total anonimato de las respuestas. Los encuestadores (médicos, fisioterapeutas y profesor de Educación Física) estuvieron presentes durante el desarrollo de las pruebas.

- El Programa de Fisioterapia, consistió en la aplicación de los agentes físicos: TENS (Transcutaneous Electrical Nerve Stimulation), onda corta capacitativa y radiación infrarroja asociada a ejercicios de movilidad articular libre sin carga; 3 veces por semana, con una hora de tratamiento, durante las 16 semanas de intervención.

- En cuanto al Programa de Educación en Salud, se desarrolló un programa de ES con 45 min de duración, una vez por semana, durante las 16 semanas de intervención, orientado a las comorbilidades frecuentes de este grupo etario y al cuidado postural, desarrollado por un profesor de Educación Física, Magíster en Actividad Física y Salud y supervisado por el equipo investigador.

- Se desarrolló igualmente un Programa de Entrenamiento de la Fuerza consistente en un protocolo de fortalecimiento para los músculos de cadera, de tipo piramidal mixto, con un entrenamiento de 3 veces por semana, durante las 16 semanas de intervención. Las cargas se incrementaron desde el 50 a $70 \%$ de la resistencia máxima $(\mathrm{RM})$ y el número de repeticiones disminuía durante las primeras 12 semanas. En las últimas 4 semanas de entrenamiento el número de repeticiones y la carga se incrementaron, hasta 75\% de la RM, a través de ejercicios analíticos, en cadena abierta, en posición supina y de pie, para los grupos musculares flexores, extensores, abductores $\mathrm{y}$ aductores de cadera, de manera bilateral y con cargas generadas a través de pesas externas autoadhesivas con velcro y plomo en tevinil. Este método de entrenamiento de la fuerza está dirigido a mejorar la coordinación intramuscular, más que provocar hipertrofia muscular ${ }^{25}$.

El programa se desarrolló sin ningún tipo de complicaciones y una vez finalizado los adultos mayores volvían a su rutina. Al finalizar el programa se agradecía a todos los participantes su colaboración.

\section{d) Análisis de los datos}

Se utilizó el programa SPSS 20.0 para Windows. El índice de participación fue de 100\% con ningún abandono durante el desarrollo; las variables cuantitativas se describen en términos de las medias aritméticas. Para ver las diferencias intra grupo se compararon las medias obtenidas antes y después de la intervención utilizando el test de Wilcoxon. Finalmente, se determinaron diferencias entre los resultados de los tres grupos post intervención, a través del estadístico KruskalWallis.

\section{Resultados}

En la Tabla 1 se muestran características de las personas estudiadas. Para todas las variables evaluadas se encontró equivalencia inicial para el test pararse y sentarse, la prueba Timed up and go y SF 36, tanto para componentes físicos como mentales Tabla 2.

Hubo mejorías significativas en los tres grupos, después de la intervención (Tabla 3). Para el test de pararse y sentarse los resultados arrojaron diferencias estadísticamente significativas $(\mathrm{p}=0,002)$ e igualmente para la prueba Timed up and go $\mathrm{p}<0,001$; por el contrario, para los resultados del SF 36v. 2.0 tanto para componentes físicos como mentales no se encontraron diferencias post inter- 
Adultos Mayores y Programas de Entrenamiento - C. E. Jiménez et al

Tabla 1. Características de los pacientes estudiados, según grupo

\begin{tabular}{|lcccc|}
\hline Parámetros & $\begin{array}{c}\text { Grupo control } \\
\mathbf{n = 1 0}\end{array}$ & $\begin{array}{c}\text { Grupo 1 } \\
\mathbf{n = 1 0}\end{array}$ & $\begin{array}{c}\text { Grupo 2 } \\
\mathbf{n = 1 0}\end{array}$ & $\begin{array}{c}\text { Total } \\
\mathbf{n = 3 0}\end{array}$ \\
Edad (años; M, DE) & $70,8(4,4)$ & $69,4(5,1)$ & $72,1(4,9)$ & 70,7 \\
Test pararse-sentarse (repeticiones) & 10,8 & 12,1 & 10,2 & 11,03 \\
Timed up and go (segundos; M, DE) & $17,6(3,4)$ & $18,4(2,5)$ & $21(2,5)$ & 19 \\
Componentes físicos (puntos) & 60,4 & 60,1 & 59,1 & 59,8 \\
Componentes mentales (puntos) & 19,6 & 18,7 & 18,6 & 18,96 \\
\hline
\end{tabular}

$\mathrm{M}=$ media; $\mathrm{DE}=$ desviación estándar.

Tabla 2. Distribución de las equivalencias en las tres pruebas intergrupos $\left(p \leq 0,05^{* *}\right)$

\begin{tabular}{|lcc|}
\hline & \multicolumn{2}{c|}{$\begin{array}{c}\text { Intervalos de confianza } \\
\text { de 99\% }\end{array}$} \\
Pruebas & $\begin{array}{c}\text { Límite } \\
\text { inferior }\end{array}$ & $\begin{array}{c}\text { Límite } \\
\text { superior }\end{array}$ \\
\hline Test pararse-sentarse & 0,0708 & 0,0846 \\
\hline Timed up and go & 0,46 & 0,4858 \\
Componentes físicos & 0,5791 & 0,6045 \\
Componentes mentales & 0,3432 & 0,3678 \\
\hline
\end{tabular}

vención ( $\mathrm{p}=0,470$ y $\mathrm{p}=0,552$, respectivamente).

La Tabla 4 muestra las medias aritméticas de las variables evaluadas en los tres grupos, antes y después de la intervención: En el test pararsesentarse y en el Time up and go la aplicación de los programas provoca cambios con respecto al grupo control, en los grupos 1 y 2; no sucediendo esto en la prueba SF 36v.2.0.

Debe señalarse que no se detectaron ninguna complicación o lesión durante la implementación del programa de fisioterapia ni durante el entrenamiento de fuerza.

Tabla 3. Pruebas Estadísticas para los test analizados $\left(p \leq 0,05^{* *}\right)$

\begin{tabular}{|lcccccc|}
\hline Estadísticos de contraste & $\boldsymbol{\chi}^{\mathbf{2}}$ & $\mathbf{g l}$ & $\begin{array}{c}\text { Sig. } \\
\text { Asintot. }\end{array}$ & $\begin{array}{c}\text { Intervalos de confianza de 99\% } \\
\text { Límite inferior }\end{array}$ & $\begin{array}{c}\text { Sig. } \\
\text { Límite superior }\end{array}$ \\
Montecarlo \\
Test pararse-sentarse post & 11,8857 & 2 & $0,002^{*}$ & 0,0004 & 0,0022 & 0,0013 \\
Timed up and go post & 20,1845 & 2 & $0^{*}$ & 0 & 0,0005 & 0 \\
Componentes físicos post & 1,5097 & 2 & 0,470 & 0,4732 & 0,4990 & 0,4861 \\
Componentes mentales post & 1,1880 & 2 & 0,552 & 0,5553 & 0,5809 & 0,5681 \\
\hline
\end{tabular}

Tabla 4. Distribución de las tres pruebas en pre y post en cada grupo

\begin{tabular}{|llcccccc|}
\hline Pruebas & & \multicolumn{2}{c}{ Grupo } & \multicolumn{2}{c}{ Grupo 1 } & \multicolumn{2}{c|}{ Grupo control } \\
Media & De & Media & De & Media & De \\
Test pararse-sentarse & Post & 14,9 & 2,60 & 15,9 & 1,60 & 12,5 & 0,97 \\
& Pre & 10,8 & 2,90 & 12,1 & 1,37 & 10,2 & 1,32 \\
Timed up and go & Post & 8,6 & 1,84 & 9,5 & 2,22 & 16,1 & 0,99 \\
& Pre & 17,6 & 3,47 & 18,4 & 2,50 & 21 & 2,49 \\
Componentes físicos & Post & 63,9 & 2,13 & 64,8 & 1,62 & 64,4 & 1,43 \\
& Pre & 60,4 & 3,47 & 60,1 & 2,23 & 59,1 & 2,42 \\
Componentes mentales & Post & 24,7 & 1,83 & 24,8 & 1,48 & 24,2 & 1,23 \\
& Pre & 19,6 & 2,01 & 18,7 & 1,70 & 18,6 & 1,78 \\
\hline
\end{tabular}




\section{Discusión}

Trabajos similares ${ }^{26,27}$ mostraron efectos positivos en programas de ejercicio de fortalecimiento en adultos mayores con artrosis de rodilla, sobre variables de fuerza muscular cuadricipital, velocidad y disminución de dependencia; misma evidencia hallada en un ensayo aleatorio controlado en pacientes con artrosis leve a moderada de cadera o rodilla, sometidos a ejercicios de fortalecimiento durante 6 semanas, además de un programa de educación en salud encontrando efectos positivos en las variables dolor, fuerza de cuádriceps, calidad de vida y disminución de visitas al fisioterapeuta.

$\mathrm{Al}$ analizar los momentos pre y post en nuestro grupo control, se identificaron diferencias significativas post tratamiento, motivadas por los efectos beneficiosos del tratamiento fisioterapéutico en la funcionalidad general. Concordamos con autores que encontraron mejorías significativas en variables de funcionalidad y riesgo de caídas en pacientes con artrosis de rodillas sometidos a un tratamiento de estimulación eléctrica transcutá$n^{2} \mathrm{a}^{28} \mathrm{y}$ con la recomendación del uso de termoterapia para disminuir la rigidez articular en la artrosis de cadera ${ }^{18}$. Nuestros hallazgos coinciden plenamente con revisiones sistemáticas de ensayos aleatorios controlados que muestran efectividad de las corrientes tipo TENS para el dolor osteoarticular, asociado a una mejoría en fuerza muscular $y$ pruebas funcionales ${ }^{29} \mathrm{y}$ el uso de termoterapia superficial y profunda con los mismos resultados ${ }^{30}$.

Para la variable calidad de vida, valorada a través del cuestionario SF 36 v2.0, encontramos diferencias post tratamiento para los componentes físicos y psicológicos, que se pueden atribuir al control del dolor obtenido con el uso de agentes físicos.

Coincidimos así con hallazgos favorables a los tratamientos termo terapéuticos y electro analgésicos en pacientes con osteoartritis, en los cuales se ha estudiado calidad de vida relacionada a la Salud, utilizando también el instrumento SF $36^{31,32}$.

Respecto del grupo 1, atribuimos las diferencias observadas al éxito que provoca instaurar un programa de Educación en Salud, sobre funcionalidad y calidad de vida, coincidiendo con otras publicaciones $^{11-13}$, respecto de los programas de Educación en Salud.

El grupo 2 obtuvo las mayores diferencias post tratamiento en las variables fuerza muscular fun- cional y riesgo de caídas, por lo que el programa de fortalecimiento, agregado al de educación en salud fue efectivo en estos pacientes, tal cual lo sugieren diversos estudios de entrenamiento de la fuerza en pacientes con artrosis ${ }^{33-35}$.

Otras investigaciones han demostrado también la eficacia de los programas de fortalecimiento y entrenamiento físico en adultos mayores ${ }^{36-38}$.

La principal limitación de nuestro estudio radica en el escaso número de participantes, debido a no existir más pacientes en este centro.

\section{Conclusiones}

- Se encontró mejoría en los tres grupos tras la aplicación de los programas respecto a lo encontrado en el pre.

- En el test pararse-sentarse y en el Time up and go la aplicación de los programas de educación para la salud y fortalecimiento muscular provocó cambios con respecto al grupo control, no sucediendo esto con los componentes físicos y mentales del SF36v.2.0, donde los tres grupos obtuvieron las mismas mejoras.

- Muchos de los problemas relacionados con los adultos mayores, son producidos por múltiples causas y complicaciones y el estado basal de los adultos mayores (pre intervención) reflejó alteraciones en equilibrio y fuerza muscular, indicando que estos elementos deben ser tratados en forma interdisciplinaria.

\section{Referencias}

1. Bazo M. Envejecimiento poblacional y el reto de la dependencia. Valencia: Nau Libres; 2011.

2. Alonso P, Sansó FJ, Díaz-Canel AM, Carrasco M, Oliva T. Envejecimiento poblacional y fragilidad en el adulto mayor. Rev Cubana Salud 2007; 33 (1).

3. Marín P. Manual de Geriatría y Gerontología. Santiago: Pontificia Universidad Católica de Chile 2000.

4. Carrasco M, Martínez G, Foradori A, Hoyl T, Valenzuela E, Quiroga T. et al. Identificación y caracterización del Adulto Mayor Saludable. Rev Med Chile 2010; 138 (9): 1077-83.

5. Salinas C, Bello M, Flores A, Carbullanca L, Torres M. Actividad física integral con adultos y adultos mayores en Chile: resultados de un programa piloto. Rev Chil Nutr 2005; 32 (3): 215-24. 
6. Zurita F, Fernández R, Cepero M, Zagalaz ML, Valverde M, Ramírez P. The relations between pain and physical activity in older adults that begin a program of physical activity. J Human Sport Exercise 2009; 4 (3): 284-97.

7. Gac H. Caídas en el Adulto Mayor. Boletín de la Escuela de Medicina Pontificia Universidad Católica de Chile 2000; 29: 71-4.

8. Deyle G, Henderson E, Metakel R, Ryder M. Efectiveness of Manual Pshycal Therapy and Exercise in Osteoarthritis of the knee. Ann Intern Med 2000; 132 (3): 173-81.

9. Acosta R. ¿Artritis o artrosis? Rev Cubana Med Gen Integ 2008; 24 (3): 1-3.

10. Ministerio de Sanidad y Política Social. Nuevas Miradas sobre el Envejecimiento. Madrid: Instituto de Mayores y Servicios Sociales-IMSERSO 2009.

11. Tamayo M, Rebolledo J. Garantía de oportunidad, artrosis y autorreporte de salud en personas con artrosis en una comuna de la Región Metropolitana. Rev Med Chile 2011; 139 (12): 1617-23.

12. Mazzuca S, Brandt K, Katz B. Effects of self-care education on the health status of inner-city patients with osteoarthritis of the knee. Arthritis Rheum 1997; 40: 1466-74.

13. Morgado A, Pérez M, Moguel F, Pérez P, Torres L. Guía de manejo clínico de la artrosis de cadera y rodilla. Rev Soc Esp Dolor 2005; 12 (5): 289-302.

14. Van-Baar M, Dekker J, Oostendorp R. The effectiveness of exercise therapy in patients with osteoarthritis of the hip or knee: a randomized clinical trial. J Rheumatolol 1998; 25: 2432-39.

15. Vilar E, Sureda S. Fisioterapia del Aparato Locomotor. Madrid: Mc Graw-Hill, Interamericana 2005.

16. Roos E, Dahlberg, L. Positive effects of moderate exercise on glycosaminoglycan content in knee cartilage. A fourmonth, randomized, controlled trial in patientes at risk of osteoarthritis. Arthritis Rheum 2005; 52: 3507-14.

17. Beyerman KL, Palmerino MB, Zohn LE, Kane GM, Forester KA. Efficacy of treating low back pain and dysfunction secondary to osteoarthritis: chiropractic care compared with moist heat alone. J Manipulative Physiol Ther 2006; 29: 107-14.

18. Abramson S, Altman RD, Arden N, Bierma-Zeinstra $\mathrm{M}$, Brandt KD, Croft P, et al. Recommendations for the management of hip and knee osteoarthritis. Osteoarthritis and Cartilage 2008; 16 (2): 137-62.

19. Más R, Mendoza S, Noa M, Valle M. Osteoartritis. Fisiopatología y tratamiento, Revista CENIC Ciencias Biológicas 2011; 42 (2): 81-8.

20. Grace C, Vance C, Rakel B, Bloggett N, De Santana J, Amendola A, et al. Effects of Transcutaneous Electrical Nerve Stimulation on Pain, Pain Sensitivity, and Function in People with Knee Osteoarthritis: A Randomized Controlled Trial. Phys Ther 2012: 92 (7): 898-910.

21. Sociedad Española de Reumatología. Artrosis: Fisiopatología, Diagnóstico y Tratamiento. Madrid: Panamericana 2010.

22. Rikli R, Jessie C. Development and validation of a functional fitness test for community-residing older adults. JAPA 1999; 7 (2): 155-60.

23. EMPAM (Examen de Medicina Preventiva del Adulto Mayor). En www.minsal.gob.cl/url;2010.

24. Olivares-Tirado P. Estado de Salud de Beneficiarios del Sistema de Salud de Chile, 2004-2005. Gobierno de Chile. Superintendencia de Isapres, Departamento de Estudios y Desarrollo Santiago de Chile: Ministerio de Salud 2007.

25. Zhelyazkov T. Bases del Entrenamiento Deportivo. Barcelona: Paidotribo 2001.

26. Fisher N, Pendergast D, Gresham G, Calkins E. Muscle rehabilitation: its effects on muscular and functional performance on patiens with knee osteoarthritis. Arch Phys Med Rehabil 1991; 72: 367-74.

27. Hopman-Rock M, Westhoff $M$. The effects of a health educational and exercise program for older adults with osteoarthtritis of the hip or knee. J Rheumatol 2000; 27: 1947-54.

28. Law P, Cheing G. Tsui A. Does transcutaneous electrical nerve stimulation improve the physical performance of people with knee osteoarthritis. J Clin Rheumatol 2004; 10 (6): 295-99.

29. Osiri M, Welch V, Brosseau L, Shea B, McGowan J, Tugwel P, et al. Estimulación eléctrica nerviosa transcutánea para la osteoartritis de rodilla. En: Biblioteca Cochrane Plus, número 3. Oxford 2008.

30. Cajigas J, Ariza R, Espinosa R, Méndez C, Mirassou $\mathrm{M}$, Robles $\mathrm{M}$, et al. Guía de práctica clínica basada en la evidencia para el diagnóstico y tratamiento de la osteoartritis. Med Int Mex 2011; 27 (6): 552-72.

31. Dios-Sancho EP, Martín-Nogueras AM. Influencia de la fisioterapia sobre el estado de salud y la calidad de vida del paciente reumático. Reumat Clin 2011; 7 (4): 224-29.

32. Rogind H, Bilbow-Nielsen B, Bodil P, Hans C. The effects of a physical training on patients with osteoarthritis of his knees. Arch Phys Med Rehabil 1998; 79: 1421-27.

33. Mangione KM, Gloviak A, Lefebvre I, Hoffman M, Craik R. The effects of high-intensity and low intensity cycle ergometry in older adults with knee osteoarthritis. J Gerontol 1999; 54 (4): 184-90.

34. Roddy E. Evidence-based recommendations for the 
role of exercise in the management of osteoarthritis of the hip or knee. The MOVE consensus. Rheumatology 2005; 44: 67-73.

35. Fiatarone-Singh M. Body Composition and weight control in older adults. Persperctives in Exercise, Nutrition and Weight Control. (Perspectives in Exercise Science and Sports Medicine) 1998; 11:243-81.

36. Cavani V, Mier C, Musto A, Tummers N. Effects of a 6-weeks resistance-training program on functional fitness of older adults. J Aging Phys Act 2002; 10: 443 52.

37. Topp R, Boardley D, Morgan AL, Fahlman M, McNevin $\mathrm{N}$. Exercise and functional task among adults who are functionally limited. West J Nurs Res 2005; 27 (3): 25270.

38. Fransen M, Mc-Connel S. Land-based Exercise for Osteoarthritis of the Knee: A metaanalysis of randomized controlled trials. J Rheumatol 2009; 36 (3): 1109-17. 